\title{
On Quadratic Unbiased Estimator for Variance Components of One-Way Repeated Measurements Model
}

\author{
Jasim Mohammed Ali Al-Isawi ${ }^{a}$, Abdulhussein Saber Al-Mouel ${ }^{b}$ \\ a Mathematics Department, College of Education for Pure Science Basrah University-Iraq .Email: jasim.ali@uobasrah.edu.iq \\ ${ }^{b}$ Mathematics Department, College of Education for Pure Science Basrah University-Iraq.Email: abdulhusseinsaber@yahoo.com
}

\section{A R T ICLE IN F O}

\section{Article history:}

Received: 17 /04/2019

Rrevised form: $00 / 00 / 0000$

Accepted : $12 / 05 / 2019$

Available online: $30 / 05 / 2019$

Keywords:

Quadratic Estimator, Repeated Measurements Model, Minimum Norm Quadratic Unbiased Estimator (MINQUE), MINQUE (1), Variance

Components, Quadratic Form, Estimation, Moore-Penrose generalized inverse.

\begin{abstract}
A B S T RAC T
In this paper, we investigate the estimator of variance components of one-way repeated measurements model (RMM) using MINQUE-principle (Rao 1971a and Rao 1971b) and method of MINQUE (1) which using priori values for components of variance.
\end{abstract}

Corresponding author Jasim Mohammed Ali Al-Isawi

Email addresses: jasim.ali@uobasrah.edu.iq

Communicated by Qusuay Hatim Egaar 


\section{Introduction}

A models of repeated measurements (RMM) are widespread in statistical studies (life, health, social, agricultural and others). And since the study of estimating the components of variance is of great importance in statistical studies, there are many statistical methods for estimating these components. The interclass correlation model is a special case of repeated measurements model introduced by Wilks (1946). Vonesh and Chinchlli (1997) introduce univariate repeated measurements Model (called One-Way Repeated Measurement Model). AL-Mouel (2004) studied the multivariate repeated measurements models and comparison of estimators. Al-Mouel A. H. S. eand others (2017) studied Bayesian One- Way Repeated Measurements Model Based on Bayes Quadratic Unbiased Estimator. Al-Isawi JA. M. A. and Al-Mouel A. H. S. (2018) studied Best Quadratic Unbiased Estimator for Variance Component of One-Way Repeated Measurements Model, in this article we study the quadratic unbiased estimator for variance components of one-way repeated measurements model. Now we introduce some definitions and remarks which used in this article.

Definition 1 [7]: For given matrix $\Lambda$ of size $n \times m$ we called a matrix $\Lambda^{+}$of size $m \times n$ is Moore-Penrose generalized inverse (MP-inverse) of $\Lambda$ if satisfy the following conditions
(a) $\Lambda \Lambda^{+} \Lambda=\Lambda$,
(b) $\Lambda^{+} \Lambda \Lambda^{+}=\Lambda^{+}$,
(c) matrix $\Lambda \Lambda^{+}$is symmetric
(d) matrix $\Lambda^{+} \Lambda$ is symmetric.

Definition 2 [7]: The Kronecker product $(\otimes)$ of an $n \times m$ and $p \times q$ matrix $A$ and $B$, is denoted by $A \otimes B$. This is an $n p \times m q$ matrix with the $(i, j)$ block $A_{i j} B$, where $i=1, \ldots, n$ and $j=1, \ldots, m$.

Kronecker product have the following properties:

1- $(A \otimes B)(D \otimes C)=A D \otimes B C$

2- $(A+B) \otimes C=(A \otimes C)+(B \otimes C)$ and $A \otimes(B+C)=(A \otimes B)+(A \otimes C)$

3- $(A \otimes B)^{+}=A^{+} \otimes B^{+}$and $(A \otimes B)^{\prime}=A^{\prime} \otimes B^{\prime}$

4- $\left(c_{1} A\right) \otimes\left(c_{2} B\right)=\left(c_{1} c_{2}\right)(A \otimes B)$

5- $\quad A \otimes\left[B_{1} \vdots B_{2}\right]=\left[A \otimes B_{1} \vdots A \otimes B_{2}\right]$

6- $\operatorname{tr}(A \otimes B)=\operatorname{tr}(A) \operatorname{tr}(B)$.

Remark 1: If $j_{p}$ denotes to $p \times 1$ vector of $1^{\prime} s, J_{p}$ denotes to the $p \times p$ matrix of $1^{\prime} s$ and $I_{p}$ denotes to $p \times p$ identity matrix then

1- $j_{p}^{+}=\frac{1}{p} j_{p}^{\prime}$.

2- $J_{p}^{+}=\frac{1}{p^{2}} J_{p}=\left(\frac{1}{p} j_{p}\right)\left(\frac{1}{p} j_{p}^{\prime}\right)$.

3- $\left(I_{p}+J_{p}\right)^{+}=I_{p}-\frac{1}{p+1} J_{p}$.

4- $\left(I_{p}-\frac{1}{p} J_{p}\right)^{+}=\left(I_{p}-\frac{1}{p} J_{p}\right)$.

Remark 2: If $A$ is any square matrix of size $q \times q$, then

$$
\left(I_{q} \otimes j_{p}\right) A\left(I_{q} \otimes j_{p}^{\prime}\right)=A \otimes J_{p}
$$

Remark 3: If $H_{p}$ denotes to $p \times p$ Idempotent matrix and $A$ any matrix, then

1- $\left(A \otimes H_{p}\right)^{+}=A^{+} \otimes H_{p}$.

2- $\left(H_{p} \otimes A\right)^{+}=H_{p} \otimes A^{+}$

3- $H_{p}^{+}=H_{p}$

4- $Y^{+}=\frac{Y^{\prime}}{Y^{\prime} Y}$ for any $n \times 1$ vector $Y$. 


\section{The one-way repeated measurements model}

Consider the following linear model and parameterization for the one-way repeated measurement model with one between-units factor incorporating univariate random effects.

$$
Y_{i j k}=\mu+\tau_{j}+\delta_{i(j)}+\gamma_{k}+(\tau \gamma)_{j k}+\varepsilon_{i j k}
$$

where

$i=1, \ldots, n$ is an index for an experimental unit within group $j$,

$j=1, \ldots, q$ is an index for levels of the between-units factor (group),

$k=1, \ldots, p$ is an index for levels of the within-units factor (time),

$Y_{i j k}$ is the response measurements at time $k$ for unit $i$ within group $j$,

$\mu$ is the overall mean,

$\tau_{j}$ is the added effect for treatment group $j$,

$\delta_{i(j)}$ is the random effect due to experimental unit $i$ within treatment group $j$,

$\gamma_{k}$ is the added effect for time $k$,

$(\tau \gamma)_{j k}$ is the added effect for the group $j \times$ time $k$ interaction, and

$\varepsilon_{i j k}$ is the random error on time $k$ for unit $i$ within group $j$.

For the parameterization to be of full rank, we impose the following set of conditions:

$$
\left.\begin{array}{l}
\sum_{j=1}^{q} \tau_{j}=0, \quad \sum_{k=1}^{p} \gamma_{k}=0, \\
\sum_{j=1}^{q}(\tau \gamma)_{j k}=0 \quad \forall k=1, \ldots, p, \quad \sum_{k=1}^{p}(\tau \gamma)_{j k}=0 \forall j=1, \ldots, q,
\end{array}\right\}
$$

We assume that the $\varepsilon_{i j k}$ 's and the $\delta_{i(j)}$ 's are independent with

$$
\varepsilon_{i j k} \sim^{i . i . d} N\left(0, \sigma_{\varepsilon}^{2}\right) \text { and } \delta_{i(j)} \sim^{i . i . d} N\left(0, \sigma_{\delta}^{2}\right)
$$

We can write model (1) as follows

$$
Y=X \beta+Z \delta+\varepsilon,
$$

Where $Y$ is $n q p$-dimensional response vector,

$Z:$ is a $n q p \times n q$ design matrix,

$\beta$ : is a $(q+1)(p+1)$-dimensional vector of fixed effects parameters,

$\delta:$ is a $n q$-dimensional vector of random effects,

$\varepsilon$ : is error term has length $n q p$ with $\varepsilon \sim N_{n q p}\left(0_{n q p}, \sigma_{\varepsilon}^{2} I_{n q p}\right)$.

And design matrix $X$ of size $n q p \times(q+1)(p+1)$ is 
$\left.\begin{array}{l}\quad X=[x 1: x 2: x 3: x 4]_{n q p \times(q p+q+p+1)} \\ \text { where } \\ \qquad 1=j_{n q p}, \quad x 2=j_{n} \otimes I_{q} \otimes j_{p}, \quad x 3=j_{n q} \otimes I_{p}, \quad x 4=j_{n} \otimes I_{q p}\end{array}\right\}$

Then from (3)

$$
\left.\begin{array}{l}
\left.\varepsilon \sim N_{n q p}\left(0_{n q p}, \sigma_{\varepsilon}^{2} I_{n q p}\right), \delta \sim N_{n q}\left(0_{n q}, \sigma_{\delta}^{2} I_{n q}\right) \text { and } \operatorname{cov}(\varepsilon, \delta)\right)=0 \\
Y \sim N_{n q p}(X \beta, \Sigma) \text { where, } \Sigma=\sigma_{\delta}^{2} Z Z^{\prime}+\sigma_{\varepsilon}^{2} I_{n q p}
\end{array}\right\}
$$

$*(\Sigma=\operatorname{var}(Y)$ is variance-covariance matrix $)$.

Lemma 1 [13]: Let $\Lambda^{+}$be MP-inverse of $\Lambda$ and put $X_{i . j}=\left(I_{n}-E_{j}\right) X_{i}$, where

$X_{i}$ is a given $n \times m_{i}$ matrix and $E_{j}=X_{j} X_{j}^{+}$. Then with $X=\left[X_{1} \vdots X_{2}\right]$

and $E_{i . j}=X_{i . j} X_{i . j}^{+}$we have $X X^{+}=E_{1}+E_{2.1}=E_{2}+E_{1.2}$.

Proposition 1: For model (4) and using Lemma 1; If $X=\left[X_{1}: X_{2}\right]$, where $X_{1}$ and $X_{2}$

are a matrix of size $n q p \times(q+1)(p+1)-m$ and $n q p \times m, 1 \leq m<(q+1)(p+1)$ then

$$
E_{2}= \begin{cases}\left(J_{n} / n\right) \otimes I_{q p} & ; m \geq q p \\ \left.J_{n} / n\right) \otimes H_{q p} & ; m<q p, H \text { is Idempotent matrix }\end{cases}
$$

and $E_{1.2}= \begin{cases}0 & ; m \geq q p \\ \left.U_{n} / n\right) \otimes\left(I_{q p}-H_{q p}\right) & ; m<q p, H=H^{2}\end{cases}$

$$
\rightarrow \quad X X^{+}=\frac{J_{n} \otimes I_{q p}}{n}
$$

\section{Proof:}

$$
\begin{aligned}
E_{2}= & X_{2} X_{2}^{+} \\
= & \left(j_{n} \otimes \chi\right)\left(j_{n}^{+} \otimes \chi^{+}\right) \text {where } \chi \text { is a matrix of size } q p \times m \\
& =j_{n} j_{n}^{+} \otimes \chi \chi^{+}= \begin{cases}\left(J_{n} / n\right) \otimes I_{q p} & ; m \geq q p \\
\left(J_{n} / n\right) \otimes H_{q p} & ; m<q p, H \text { is Idempotent matrix }\end{cases}
\end{aligned}
$$

Similarly $E_{1}=X_{1} X_{1}^{+}= \begin{cases}\left(J_{n} / n\right) \otimes I_{q p} & ; m<q p \\ \left(J_{n} / n\right) \otimes H_{q p} & ; m \geq q p, H \text { is Idempotent matrix }\end{cases}$

And $E_{1.2}=X_{1.2} X_{1.2}^{+}=\left[\left(I_{n q p}-E_{2}\right) X_{1}\right]\left[\left(I_{n q p}-E_{2}\right) X_{1}\right]^{+}$

$$
\begin{aligned}
& =\left(I_{n q p}-E_{2}\right) X_{1} X_{1}^{+}\left(I_{n q p}-E_{2}\right) \\
& = \begin{cases}\left.\left.\left(I_{n}-\left(J_{n} / n\right)\right) \otimes I_{q p}\right)\left(\left(J_{n} / n\right) \otimes H_{q p}\right)\left(I_{n}-\left(J_{n} / n\right)\right) \otimes I_{q p}\right) & ; m \geq q p \\
\left.\left(I_{n q p}-\left(J_{n} / n\right) \otimes H_{q p}\right)\left(U_{n} / n\right) \otimes I_{q p}\right)\left(I_{n q p}-\left(J_{n} / n\right) \otimes H_{q p}\right) & ; m<q p\end{cases} \\
& = \begin{cases}\left(I_{n}-\left(J_{n} / n\right)\right)\left(J_{n} / n\right)\left(I_{n}-\left(J_{n} / n\right)\right) \otimes H_{q p} \quad ; m \geq q p \\
\left.J_{n} / n\right) \otimes\left(I_{q p}-H_{q p}\right) & ; m<q p\end{cases} \\
& = \begin{cases}\left.\left(J_{n} / n\right)-\left(J_{n} / n\right)\right) \otimes H_{q p}=0 & ; m \geq q p \\
\left.J_{n} / n\right) \otimes I_{q p}-\left(J_{n} / n\right) \otimes H_{q p} & ; m<q p\end{cases}
\end{aligned}
$$

15

$\rightarrow E_{1.2}=\left\{\begin{array}{lll}0 & ; m \geq q p \\ \left(J_{n} / n\right) \otimes\left(I_{q p}-H_{q p}\right) & m<q p, \quad \mathrm{H}=\mathrm{H}^{2}\end{array}\right.$

$\rightarrow X X^{+}=E_{2}+E_{1.2}=\frac{J_{n} \otimes I_{q p}}{n}$ 
It is clear that

$$
\begin{aligned}
X & =\left[j_{n q p} \vdots j_{n} \otimes I_{q} \otimes j_{p} \vdots j_{n q} \otimes I_{p} \vdots j_{n} \otimes I_{q p}\right] \\
& =j_{n} \otimes\left[j_{q p} \vdots I_{q} \otimes j_{p} \vdots j_{q} \otimes I_{p} \vdots I_{q p}\right] \\
& =\left(j_{n} \otimes \chi\right) \text { where } \chi=\left[j_{q p} \vdots I_{q} \otimes j_{p} \vdots j_{q} \otimes I_{p} \vdots I_{q p}\right]_{q p \times(q+1)(p+1)} \\
\rightarrow X X^{+} & =\left(j_{n} \otimes \chi\right)\left(j_{n}^{+} \otimes \chi^{+}\right) \\
& =j_{n} j_{n}^{+} \otimes \chi \chi^{+} \quad\left(j_{n}^{+}=\frac{j_{n}^{\prime}}{n} \quad \text { and } \chi \chi^{+}=I_{q p}\right) \\
& =\frac{J_{n} \otimes I_{q p}}{n}
\end{aligned}
$$

Complete proof.

Proposition 2: For model (4) and using Lemma 1, with $U=\left[U_{1} \vdots U_{2}\right]$ where $U_{1}=X: U_{2}=Z$, then we have $E_{1}=U_{1} U_{1}^{+}=X X^{+}=\frac{J_{n} \otimes I_{q p}}{n}$

$$
\text { and } \quad E_{2.1}=U_{2.1} U_{2.1}^{+}=\frac{I_{n q} \otimes J_{p}}{p}-\frac{J_{n} \otimes I_{q} \otimes J_{p}}{n p}=\frac{\left(I_{n}-\left(J_{n} / n\right)\right) \otimes I_{q} \otimes J_{p}}{p}
$$

Implies to

\section{Proof:}

$$
U\left(U^{\prime} U\right)^{+} U^{\prime}=U U^{+}=\frac{J_{n} \otimes I_{q p}}{n}+\frac{\left(I_{n}-\left(J_{n} / n\right)\right) \otimes I_{q} \otimes J_{p}}{p}
$$

Since $U_{2.1}=\left(I_{n q p}-E_{1}\right) Z, Z=I_{n q} \otimes j_{p}$ and $E_{1}=\frac{J_{n} \otimes I_{q p}}{n}($ proved in Proposition 1$)$.

$$
\begin{aligned}
\therefore \quad U_{2.1} & \left.=\left(I_{n q p}-\left(J_{n} / n\right)\right) \otimes I_{q p}\right) Z \\
U_{2.1} & =\left(\left(I_{n}-\left(J_{n} / n\right)\right) \otimes I_{q p}\right) Z \\
\rightarrow \quad E_{2.1} & =U_{2.1} U_{2.1}^{+}=\left[\left(\left(I_{n}-\left(J_{n} / n\right)\right) \otimes I_{q p}\right) Z\right]\left[\left(\left(I_{n}-\left(J_{n} / n\right)\right) \otimes I_{q p}\right) Z\right]^{+} \\
& =\left(\left(I_{n}-\left(J_{n} / n\right)\right) \otimes I_{q p}\right)\left(Z Z^{+}\right)\left(\left(I_{n}-\left(J_{n} / n\right)\right) \otimes I_{q p}\right)^{+} \\
& =\left(\left(I_{n}-\left(J_{n} / n\right)\right) \otimes I_{q p}\right)\left(\left(I_{n q} \otimes j_{p}\right)\left(I_{n q} \otimes j_{p}\right)^{+}\right)\left(\left(I_{n}-\left(J_{n} / n\right)\right) \otimes I_{q p}\right)^{+} \\
& =\left(\left(I_{n}-\left(J_{n} / n\right)\right) \otimes I_{q p}\right)\left(I_{n q} \otimes J_{p} / p\right)\left(\left(I_{n}-\left(J_{n} / n\right)\right) \otimes I_{q p}\right) \\
& =\left(\left(I_{n}-\left(J_{n} / n\right)\right) \otimes I_{q p}\right)\left(I_{n} \otimes I_{q} \otimes\left(J_{p} / p\right)\right)\left(\left(I_{n}-\left(J_{n} / n\right)\right) \otimes I_{q p}\right) \\
& =\left(\left(I_{n}-\left(J_{n} / n\right)\right) \otimes I_{q} \otimes I_{p}\right)\left(I_{n} \otimes I_{q} \otimes\left(J_{p} / p\right)\right)\left(\left(I_{n}-\left(J_{n} / n\right)\right) \otimes I_{q p}\right) \\
& =\left(\left(I_{n}-\left(J_{n} / n\right)\right) \otimes I_{q} \otimes\left(J_{p} / p\right)\right)\left(\left(I_{n}-\left(J_{n} / n\right)\right) \otimes I_{q} \otimes I_{p}\right) \\
& =\left(\left(I_{n}-\left(J_{n} / n\right)\right) \otimes I_{q} \otimes\left(J_{p} / p\right)\right) \\
\rightarrow \quad U U^{+} & =E_{1}+E_{2.1}=\frac{J_{n} \otimes I_{q p}}{n}+\frac{\left(I_{n}-\left(J_{n} / n\right)\right) \otimes I_{q} \otimes J_{p}}{p} .
\end{aligned}
$$

Complete proof. 


\section{1- MINQUE for $\sigma_{\varepsilon}^{2}$}

Let $A$ be an $n q p \times n q p$ matrix; then a quadratic estimator for $\sigma_{\varepsilon}^{2}$ is defined as

$$
\hat{\sigma}_{\varepsilon}^{2}=Y^{\prime} A Y
$$

Note: For matrix $A$ without loss of generality, we can assume that $A$ is a symmetric matrix and nonnegative definite, to make $\hat{\sigma}_{\varepsilon}^{2}$ nonnegative for all $Y$.

When a ratio $\frac{\sigma_{\delta}^{2}}{\sigma_{\varepsilon}^{2}}$ is known which equal to $\theta$, or $\sigma_{\delta}^{2}$ and $\sigma_{\varepsilon}^{2}$ are equal $(\theta=1)$.

We can write model (4) as follows

$$
Y=X \beta+\varepsilon,
$$

Where $\varepsilon=Z \delta+\varepsilon \rightarrow E(\boldsymbol{\varepsilon})=0, \operatorname{var}(\boldsymbol{\varepsilon})=\sigma_{\varepsilon}^{2}\left(I+\theta Z Z^{\prime}\right)$

Since $E\left(\boldsymbol{\varepsilon}^{\prime} A \boldsymbol{\varepsilon}\right)=\operatorname{tr}(A \operatorname{var}(\boldsymbol{\varepsilon}))$ and $E\left(\boldsymbol{\varepsilon}^{\prime}\right)=0$, then

$$
\begin{aligned}
E\left(\hat{\sigma}_{\varepsilon}^{2}\right) & =E\left(Y^{\prime} A Y\right)=E\left[(X \beta+\varepsilon)^{\prime} A(X \beta+\varepsilon)\right] \\
& =\beta^{\prime} X^{\prime} A X \beta+E\left(\boldsymbol{\varepsilon}^{\prime} A \boldsymbol{\varepsilon}+2 \boldsymbol{\varepsilon}^{\prime} A X \beta\right) \\
& =\beta^{\prime} X^{\prime} A X \beta+\operatorname{tr}(A \operatorname{var}(\boldsymbol{\varepsilon})) \\
& =\beta^{\prime} X^{\prime} A X \beta+\sigma_{\varepsilon}^{2} \operatorname{tr}\left(A\left(I+\theta Z Z^{\prime}\right)\right)
\end{aligned}
$$

$\rightarrow E\left(\hat{\sigma}_{\varepsilon}^{2}\right)=\beta^{\prime} X^{\prime} A X \beta+\sigma_{\delta}^{2} \operatorname{tr}\left(Z^{\prime} A Z\right)+\sigma_{\varepsilon}^{2} \operatorname{tr}(A)$

To make $\hat{\sigma}_{\varepsilon}^{2}$ unbiased that minimizes the norm of matrix $A$ must be have

$$
\|A\|^{2}=\operatorname{tr}\left(A A^{\prime}\right)=\min
$$

and $\quad X^{\prime} A X=0, Z^{\prime} A Z=0$, and $\operatorname{tr}(A)=1$.

To solve this problem let we assume that

$$
U=[X: Z] \rightarrow U^{\prime} A U=\left[\begin{array}{ll}
X^{\prime} A X & X^{\prime} A Z \\
Z^{\prime} A X & Z^{\prime} A Z
\end{array}\right]
$$

Since $X^{\prime} A X=0, Z^{\prime} A Z=0$ and $A$ is a symmetric and nonnegative matrix then

$$
A X=0 \text { and } A Z=0 \rightarrow X^{\prime} A Z=0 \text { and } Z^{\prime} A X=0
$$

From (14) and (15) we have $U^{\prime} A U=0$ which implies that problem (13) becomes

$$
\operatorname{tr}\left(A A^{\prime}\right)=\min ,
$$

under restrictions

$$
U^{\prime} A U=0 \text { and } \operatorname{tr}(A)=1
$$

To solve problems (16-17) using a Lagrange function for multiplier matrix (Lagrange multipliers technique), the Lagrange function can be defined as

$$
f(A, L, \lambda)=\frac{1}{2} \operatorname{tr}\left(A A^{\prime}\right)+\operatorname{tr}\left(U^{\prime} A U L^{\prime}\right)+(1-\operatorname{tr}(A)) \lambda
$$

where $L$ is $m \times m$ Lagrange multiplier matrix and $\lambda$ is scalar.

17 ng this formula, for any matrices $A, B, C$ of appropriate size.

$$
\frac{\partial \operatorname{tr}(A)}{\partial A}=I, \quad \frac{\partial \operatorname{tr}\left(A A^{\prime}\right)}{\partial A}=2 A, \quad \frac{\partial \operatorname{tr}(B A C)}{\partial A}=B^{\prime} C^{\prime}
$$

differentiate function $f$ with respect to $A$,

For optimization (16) under restrictions (17) is that the derivative $\partial f / \partial A$ must be equal to zero,

$$
\frac{\partial f}{\partial A}=A+U L U^{\prime}-\lambda I=0
$$

From equation (18) we have

$$
A=\lambda I-U L U^{\prime}
$$

To find $L$ and $\lambda$ in (19), we using the conditions in (17), we have 
$U^{\prime} \lambda I U-U^{\prime} U L U^{\prime} U=0 \rightarrow L=\lambda\left(U^{\prime} U\right)^{+}$

substituting the value of $L(20)$ in (19) , we have

$$
\begin{aligned}
A & =\lambda\left(I-U\left(U^{\prime} U\right)^{+} U^{\prime}\right)=\lambda\left(I-U U^{+}\right) \\
\text {and } \quad & \lambda=\frac{1}{\operatorname{rank}\left(I-U U^{+}\right)}
\end{aligned}
$$

The matrix $\left[I-U U^{+}\right]$is idempotent (proved by [6] Graybill(1983)) then

$$
\begin{gathered}
\operatorname{rank}\left[I-U U^{+}\right]=\operatorname{tr}\left(I-U U^{+}\right)=n q p-\operatorname{rank}(U) \\
\therefore \quad \lambda=\frac{1}{n q p-\operatorname{rank}(U)}, \quad \operatorname{rank}(U)=q(n+p-1) \\
\rightarrow \quad A=\frac{\left(I-U\left(U^{\prime} U\right)^{+} U^{\prime}\right)}{q(n p-n-p+1)}=\frac{\left(I-U U^{+}\right)}{q(n-1)(p-1)}
\end{gathered}
$$

From (11) and (22), we have

$$
\hat{\sigma}_{\varepsilon}^{2}=Y^{\prime} \frac{\left(I-U U^{+}\right)}{q(n-1)(p-1)} Y . \square
$$

And

$$
\hat{\sigma}_{\delta}^{2}=Y^{\prime} \frac{\theta\left(I-U U^{+}\right)}{q(n-1)(p-1)} Y . \square
$$

From Proposition 2,

Since $\quad U\left(U^{\prime} U\right)^{+} U^{\prime}=E_{1}+E_{2.1}$

we have $U\left(U^{\prime} U\right)^{+} U^{\prime}=U U^{+}=\frac{J_{n} \otimes I_{q p}}{n}+\frac{\left(I_{n}-\left(J_{n} / n\right)\right) \otimes I_{q} \otimes J_{p}}{p}$

Substituting (25) in(22) $A=\frac{\left(I_{n q p}-\left(J_{n} / n\right) \otimes I_{q p}+\left(I_{n}-\left(J_{n} / n\right)\right) \otimes I_{q} \otimes\left(J_{p} / p\right)\right)}{q(n-1)(p-1)}$

$$
\rightarrow \quad A=\frac{\left(I_{n}-\left(J_{n} / n\right)\right) \otimes I_{q} \otimes\left(I_{p}-\left(J_{p} / p\right)\right)}{q(n-1)(p-1)}
$$

From (23), (24) and (26) we have, the quadratic estimator for $\sigma_{\varepsilon}^{2}$ and $\sigma_{\delta}^{2}$ are

And

$$
\hat{\sigma}_{\varepsilon}^{2}=Y^{\prime} \frac{\left(I_{n}-\left(J_{n} / n\right)\right) \otimes I_{q} \otimes\left(I_{p}-\left(J_{p} / p\right)\right)}{q(n-1)(p-1)} Y
$$

$$
\hat{\sigma}_{\delta}^{2}=Y^{\prime} \frac{\theta\left(I_{n}-\left(J_{n} / n\right)\right) \otimes I_{q} \otimes\left(I_{p}-\left(J_{p} / p\right)\right)}{q(n-1)(p-1)} Y
$$




\section{2- MINQUE for $\sigma=\left[\sigma_{\delta}^{2}, \sigma_{\varepsilon}^{2}\right]^{\prime}$}

The derivation of the law is based on minimizing the Euclidean norm. If the mixed linear model is expressed as a matrix

For model (4) we have that $\boldsymbol{Y} \sim N\left(X \beta, \boldsymbol{\Sigma}=\sigma_{0}^{2} I_{n q p}+\sigma_{1}^{2} Z_{1} Z_{1}^{\prime}\right)$,

Such that $\sigma_{0}^{2}=\sigma_{\varepsilon}^{2}, \sigma_{1}^{2}=\sigma_{\delta}^{2}$ and $Z_{0}=I_{n q p}, Z_{1}=Z$ in model (4)

We can express the model (4) as

$$
\boldsymbol{Y}=X \beta+\boldsymbol{Z} \boldsymbol{\delta}
$$

where $\boldsymbol{Z}=\left[Z_{0} \vdots Z_{1}\right]$ and $\boldsymbol{\delta}^{\prime}=\left[\varepsilon^{\prime} \vdots \delta^{\prime}\right]$. The model (29) is called a mixed linear model. Thus generally we have $E(Y)=X \beta$ and $\boldsymbol{\Sigma}=\sigma_{0}^{2} V_{0}+\sigma_{1}^{2} V_{1}$, where $V_{r}=Z_{r} Z_{r}^{\prime}, r=0,1 . \boldsymbol{\Sigma}$ is called the covariance matrix and the parameters $\sigma_{0}^{2}, \sigma_{1}^{2}$ are the unknown components of variance whose values should be estimated.

We can write a linear combination for the components of variance $\sigma_{r}^{2} s$, by a quadratic

form $\boldsymbol{Y}^{\prime} A \boldsymbol{Y}$, where $A$ is a symmetric matrix chosen subject to the conditions which, guarantee the estimator's unbiasedness and invariance we have

$$
\text { and } \quad \begin{aligned}
E\left(\boldsymbol{Y}^{\prime} A \boldsymbol{Y}\right) & =c_{0} \sigma_{0}^{2}+c_{1} \sigma_{1}^{2}=\boldsymbol{C}^{\prime} \boldsymbol{\sigma} \\
\boldsymbol{Y}^{\prime} A \boldsymbol{Y} & =(X \beta+\boldsymbol{Z} \boldsymbol{\delta})^{\prime} A(X \beta+\boldsymbol{Z} \boldsymbol{\delta}) \\
& =\beta^{\prime} X^{\prime} A X \beta+2 \beta^{\prime} X^{\prime} A \boldsymbol{Z} \boldsymbol{\delta}+\boldsymbol{\delta}^{\prime} \boldsymbol{Z}^{\prime} A \boldsymbol{Z} \boldsymbol{\delta}
\end{aligned}
$$

Under unbiasedness and invariance, the estimator reduces to

$$
\boldsymbol{Y}^{\prime} A \boldsymbol{Y}=\boldsymbol{\delta}^{\prime} \boldsymbol{Z}^{\prime} A \boldsymbol{Z} \boldsymbol{\delta}
$$

Where $\boldsymbol{C}=\left[c_{0}, c_{1}\right]^{\prime}$ and $A$ is chosen to satisfy the restrictions

$$
A X=0 \text { and } c_{r}=\operatorname{tr}\left(Z_{r}^{\prime} A Z_{r}\right), r=0,1 .
$$

Clear that: $\boldsymbol{\delta}$ has a normal distribution (since $\varepsilon \sim i i d . N\left(0, \sigma_{0}^{2} I\right)$ and $\delta \sim i i d . N\left(0, \sigma_{1}^{2} I\right)$ ). The components of variance are a linear function of the natural estimated, so it should be $\boldsymbol{\delta}^{\prime} D \boldsymbol{\delta}$ where $D$ is known diagonal matrix.

The difference between the proposed estimator (30) and the natural unbiased estimator $\left(\boldsymbol{\delta}^{\prime} D \boldsymbol{\delta}\right)$ is

$$
\boldsymbol{\delta}^{\prime}\left(\boldsymbol{Z}^{\prime} A \boldsymbol{Z}-D\right) \boldsymbol{\delta}
$$

Remark 4: $\left\|\boldsymbol{Z}^{\prime} A \boldsymbol{Z}-D\right\|^{2}=\operatorname{tr}\left[\left(\boldsymbol{Z}^{\prime} A \boldsymbol{Z}-D\right)^{2}\right]=\min$. (Rao1971a deduced )[9].

The MINQUE method tries to find minimize the difference in (33) with the restrictions in (32).

19

aimize the square of Euclidean norm ( || ||) using (Remark 4) inasmuch

$$
\left\|\boldsymbol{Z}^{\prime} A \boldsymbol{Z}-D\right\|^{2}=\operatorname{tr}\left[\left(\boldsymbol{Z}^{\prime} A \boldsymbol{Z}-D\right)^{2}\right]=\operatorname{tr}\left[(A \boldsymbol{V})^{2}\right]-\operatorname{tr}\left[D^{2}\right]
$$

Where $\quad \boldsymbol{V}=V_{0}+V_{1}$

Inasmuch as $\operatorname{tr}\left[D^{2}\right]$ does not involve $A$, the problem of MINQUE reduces to minimizing $\operatorname{tr}\left[(A \boldsymbol{V})^{2}\right]$ with the conditions in (32) attained at, according to Rao [11]

$$
A=a_{0} Q V_{0} Q+a_{1} Q V_{1} Q
$$

Where

$$
Q=\boldsymbol{V}^{-1}\left(I-X\left(X^{\prime} \boldsymbol{V}^{-1} X\right)^{+} X^{\prime} \boldsymbol{V}^{-1}\right)
$$

And $\boldsymbol{a}=\left[a_{0}, a_{1}\right]^{\prime}$ is determined from the equations $\boldsymbol{a}=\boldsymbol{S}^{+} \boldsymbol{C}$, 
with

$$
\boldsymbol{S}=\left(S_{r, s}\right)=\operatorname{tr}\left(Q V_{r} Q V_{s}\right), r=0,1 \text { and } s=0,1 .
$$

where $X$ is the matrix in the model in (29) and $\boldsymbol{V}$ is a positive definite matrix.

For the problem of MINQUE, choosing $\boldsymbol{V}=V_{0}+V_{1}$ or $\Sigma_{(t)}=t_{0} V_{0}+t_{1} V_{1}$,

where $t=\left[t_{0}, t_{1}\right]^{\prime}$ are a priori ratios of unknown components of variance.

On using (34), we have the MINQUE of $\boldsymbol{C}^{\prime} \boldsymbol{\sigma}$ is given by

$$
\boldsymbol{C}^{\prime} \widehat{\boldsymbol{\sigma}}=\boldsymbol{Y}^{\prime} A \boldsymbol{Y}=\boldsymbol{Y}^{\prime}\left(a_{0} Q V_{0} Q+a_{1} Q V_{1} Q\right) \boldsymbol{Y}
$$

the estimator (35) can be written as

$$
\boldsymbol{C}^{\prime} \widehat{\boldsymbol{\sigma}}=\boldsymbol{a}^{\prime} \boldsymbol{\gamma}, \text { where } \boldsymbol{\gamma}=\left[\begin{array}{l}
\boldsymbol{Y}^{\prime} Q V_{0} Q \boldsymbol{Y} \\
\boldsymbol{Y}^{\prime} Q V_{1} Q \boldsymbol{Y}
\end{array}\right]
$$

On substituting $\boldsymbol{a}=\boldsymbol{S}^{+} \boldsymbol{C}$ in (36), we have

$$
C^{\prime} \hat{\sigma}=C^{\prime} S^{+} \gamma \rightarrow \widehat{\sigma}=S^{+} \gamma .
$$

The solution vector (37) is unique if and only if the individual components are unbiased.

Now since $\sigma_{0}^{2}$ and $\sigma_{1}^{2}$ not equal

$$
\text { Let } \quad \alpha_{0}=\frac{\varepsilon}{\sigma_{0}} \text { and } \quad \alpha_{1}=\frac{\delta}{\sigma_{1}}
$$

Then the difference in (33) is given by

$$
\boldsymbol{\alpha}^{\prime} \Psi^{1 / 2}\left(\boldsymbol{Z}^{\prime} A \boldsymbol{Z}-D\right) \Psi^{1 / 2} \boldsymbol{\alpha}
$$

Where $\boldsymbol{\alpha}^{\prime}=\left(\alpha_{0}^{\prime} \vdots \alpha_{1}^{\prime}\right)$ and $\Psi=\left[\begin{array}{cc}\sigma_{0}^{2} I & 0 \\ 0 & \sigma_{1}^{2} I\end{array}\right]$

Now, the minimization of (39) using (Remark 4) is equivalent to minimizing $\operatorname{tr}\left[(A \boldsymbol{\Sigma})^{2}\right]$ under the restrictions in (32),

Where $\boldsymbol{\Sigma}$ defined in (29) as.

$$
\boldsymbol{\Sigma}=\sigma_{0}^{2} V_{0}+\sigma_{1}^{2} V_{1}=\sigma_{0}^{2}\left(V_{0}+\frac{\sigma_{1}^{2}}{\sigma_{0}^{2}} V_{1}\right)
$$

The matrix $\boldsymbol{\Sigma}$ in (40) have two unknown variance $\left(\sigma_{r}^{2}, r=0,1\right)$.

Then according to Rao [10], we have two amendments to this problem:

1. If we have a priori knowledge of the approximate ratio $\frac{\sigma_{1}^{2}}{\sigma_{0}^{2}}$, we can substitute them in (40) and use the $\Sigma$ thus computed like as estimator in (section 3.1).

2. We can use a priori estimates in (40) and obtain MINQUEs of $\sigma_{r}^{2}, r=0,1$.

These estimates then may be substituted in (40) many times. The procedure is called iterative MINQUE or I-MINQUE (Rao and Kleffé,1988) [8]. In this procedure, the MINQUE estimator of the variance components can be obtained by solving the system of equations (37) 


$$
\left[\begin{array}{ll}
\operatorname{tr}\left(Q_{(t)} V_{0} Q_{(t)} V_{0}\right) & \operatorname{tr}\left(Q_{(t)} V_{0} Q_{(t)} V_{1}\right) \\
\operatorname{tr}\left(Q_{(t)} V_{1} Q_{(t)} V_{0}\right) & \operatorname{tr}\left(Q_{(t)} V_{1} Q_{(t)} V_{1}\right)
\end{array}\right]\left[\begin{array}{c}
\hat{\sigma}_{0}^{2} \\
\hat{\sigma}_{1}^{2}
\end{array}\right]=\left[\begin{array}{c}
\boldsymbol{Y}^{\prime} Q_{(t)} V_{0} Q_{(t)} \boldsymbol{Y} \\
\boldsymbol{Y}^{\prime} Q_{(t)} V_{1} Q_{(t)} \boldsymbol{Y}
\end{array}\right]
$$

Where

$$
\begin{gathered}
Q_{(t)}=\Sigma_{(t)}^{-1}\left(I-X\left(X^{\prime} \Sigma_{(t)}^{-1} X\right)^{+} X^{\prime} \Sigma_{(t)}^{-1}\right) \\
\Sigma_{(t)}=t_{0} V_{0}+t_{1} V_{1} \quad ; t=\left[t_{0}, t_{1}\right]^{\prime},
\end{gathered}
$$

Although the estimate of the variance component depends on a priori value of the human choice $t_{r}$, as long as these a priori values do not depend on the experimental data, the MINQUE estimator is still unbiased. Choose any a priori $t_{r}$, can be obtained the variance component estimate $\left[\sigma_{r}^{2}\right]$. New estimates can be obtained if the estimates are replaced with priori estimates for reevaluation value. This process is repeated until the new estimate is very close to the old estimate. This iterative estimation method is like to relative maximum likelihood estimator (REML) method, which is a result of the maximum likelihood estimate (EML). In other word, REML estimates and MINQUE estimates are relatively close. For more see [12].

\section{3- MINQUE (1) for $\sigma_{\varepsilon}^{2}$ and $\sigma_{\delta}^{2}$}

The choice of a priori $t_{r}$ in (42) can based on experience or even on past analysis. The easier way is to take it all a priori values are $1\left(t_{r}=1\right)$. This method is called the MINQUE (1) method, and the variance component obtained is estimated metering is a MINQUE (1) estimate.

The unbiased estimator of the MINQUE (1) \{MINQUE on $\left.t=j_{2}\right\}$ is,

Assume $t=j_{2}=\left[\begin{array}{l}1 \\ 1\end{array}\right]$, substituting $t_{r}$ in (42) we have

$$
\left.\begin{array}{l}
\Sigma_{(1)}=V_{0}+V_{1}=\boldsymbol{V}=Z_{0} Z_{0}^{\prime}+Z_{1} Z_{1}^{\prime}=I_{n q} \otimes\left(I_{p}+J_{p}\right), \\
Q_{(1)}=\Sigma_{(1)}^{-1}\left(I-X\left(X^{\prime} \Sigma_{(1)}^{-1} X\right)^{+} X^{\prime} \Sigma_{(1)}^{-1}\right)
\end{array}\right\}
$$

Let $U=\left(\Sigma_{(1)}^{-1}\right)^{1 / 2} X$ and $P_{U}=U\left(U^{\prime} U\right)^{+} U^{\prime}$ is projection matrix.

Then

$$
\begin{aligned}
Q_{(1)}= & \left(\Sigma_{(1)}^{-1}\right)^{\frac{1}{2}}\left(I-P_{U}\right)\left(\Sigma_{(1)}^{-1}\right)^{\frac{1}{2}} \\
Q_{(1)} X & =\left(\Sigma_{(1)}^{-1}\right)^{\frac{1}{2}}\left(I-P_{U}\right)\left(\Sigma_{(1)}^{-1}\right)^{\frac{1}{2}} X \\
& =\left(\Sigma_{(1)}^{-1}\right)^{\frac{1}{2}}\left(I-P_{U}\right) U \\
& =\left(\Sigma_{(1)}^{-1}\right)^{\frac{1}{2}}(U-U)=0
\end{aligned}
$$

Therefor $r=0,1$ and $s=0,1$ we have

$$
\begin{aligned}
E\left[\boldsymbol{Y}^{\prime} Q_{(1)} V_{r} Q_{(1)} \boldsymbol{Y}\right] & =\operatorname{tr}\left(Q_{(1)} V_{r} Q_{(1)} \boldsymbol{\Sigma}\right)+(X \beta)^{\prime} Q_{(1)} V_{r} Q_{(1)} X \beta \\
& =\operatorname{tr}\left(Q_{(1)} V_{r} Q_{(1)}\left(\sigma_{0}^{2} V_{0}+\sigma_{1}^{2} V_{1}\right)\right) \\
& =\operatorname{tr}\left(\sigma_{0}^{2} Q_{(1)} V_{r} Q_{(1)} V_{0}+\sigma_{1}^{2} Q_{(1)} V_{r} Q_{(1)} V_{1}\right)
\end{aligned}
$$

Implies to

$$
E\left[\begin{array}{l}
\boldsymbol{Y}^{\prime} Q_{(t)} V_{0} Q_{(t)} \boldsymbol{Y} \\
\boldsymbol{Y}^{\prime} Q_{(t)} V_{1} Q_{(t)} \boldsymbol{Y}
\end{array}\right]=\left[\begin{array}{ll}
\operatorname{tr}\left(Q_{(1)} V_{0} Q_{(1)} V_{0}\right) & \operatorname{tr}\left(Q_{(1)} V_{0} Q_{(1)} V_{1}\right) \\
\operatorname{tr}\left(Q_{(1)} V_{1} Q_{(1)} V_{0}\right) & \operatorname{tr}\left(Q_{(1)} V_{1} Q_{(1)} V_{1}\right)
\end{array}\right]\left[\begin{array}{c}
\sigma_{0}^{2} \\
\sigma_{1}^{2}
\end{array}\right]
$$

Where 


$$
\begin{gathered}
\boldsymbol{S}=\left[\begin{array}{ll}
\operatorname{tr}\left(Q_{(1)} V_{0} Q_{(1)} V_{0}\right) & \operatorname{tr}\left(Q_{(1)} V_{0} Q_{(1)} V_{1}\right) \\
\operatorname{tr}\left(Q_{(1)} V_{1} Q_{(1)} V_{0}\right) & \operatorname{tr}\left(Q_{(1)} V_{1} Q_{(1)} V_{1}\right)
\end{array}\right]_{2 \times 2}=\left[\begin{array}{ll}
\lambda_{00} & \lambda_{01} \\
\lambda_{10} & \lambda_{11}
\end{array}\right] \\
\text { and } \boldsymbol{\sigma}=\left[\begin{array}{c}
\sigma_{0}^{2} \\
\sigma_{1}^{2}
\end{array}\right], \lambda_{r, s}=\text { sumation all eigenvalues of } Q_{(1)} V_{r} Q_{(1)} V_{S} .
\end{gathered}
$$

we have $\quad \boldsymbol{S} \widehat{\boldsymbol{\sigma}}=\left[\begin{array}{l}\boldsymbol{Y}^{\prime} Q_{(1)} V_{0} Q_{(1)} \boldsymbol{Y} \\ \boldsymbol{Y}^{\prime} Q_{(1)} V_{1} Q_{(1)} \boldsymbol{Y}\end{array}\right]_{2 \times 1}$

$$
\hat{\sigma}^{2}=\boldsymbol{S}^{+}\left[\begin{array}{l}
\boldsymbol{Y}^{\prime} Q_{(1)} V_{0} Q_{(1)} \boldsymbol{Y} \\
\boldsymbol{Y}^{\prime} Q_{(1)} V_{1} Q_{(1)} \boldsymbol{Y}
\end{array}\right], \boldsymbol{S}^{+}=\frac{1}{|\boldsymbol{S}|}\left[\begin{array}{rr}
\lambda_{1,1} & -\lambda_{0,1} \\
-\lambda_{1,0} & \lambda_{0,0}
\end{array}\right],|\boldsymbol{S}| \text { is determain of } \boldsymbol{S}
$$

We can write as $\left[\operatorname{tr}\left(Q_{(t)} Z_{r} Z_{r}^{\prime} Q_{(t)} Z_{s} Z_{s}^{\prime}\right)\right]\left[\hat{\sigma}_{r}^{2}\right]=\left[\boldsymbol{Y}^{\prime} Q_{(t)} Z_{r} Z_{r}^{\prime} Q_{(t)} \boldsymbol{Y}\right]$

Relationships (43-46) are proof of (41). $\square$

Then the MINQUE for $\sigma_{\varepsilon}^{2}$ and $\sigma_{\delta}^{2}$ are

and

$$
\begin{aligned}
\hat{\sigma}_{\varepsilon}^{2} & =\hat{\sigma}_{0}^{2}=\boldsymbol{Y}^{\prime}\left(s_{0,0}^{+} Q_{(1)} Z_{0} Z_{0}^{\prime} Q_{(1)}+s_{0,1}^{+} Q_{(1)} Z_{1} Z_{1}^{\prime} Q_{(1)}\right) \boldsymbol{Y} . \\
& =\boldsymbol{Y}^{\prime}\left[\frac{1}{|\boldsymbol{S}|}\left(\lambda_{1,1} Q_{(1)} Q_{(1)}-\lambda_{0,1} Q_{(1)}\left(I_{n q} \otimes J_{p}\right) Q_{(1)}\right)\right] \boldsymbol{Y} .
\end{aligned}
$$

$$
\begin{aligned}
\hat{\sigma}_{\delta}^{2} & =\hat{\sigma}_{1}^{2}=\boldsymbol{Y}^{\prime}\left(s_{1,0}^{+} Q_{(1)} Z_{0} Z_{0}^{\prime} Q_{(1)}+s_{1,1}^{+} Q_{(1)} Z_{1} Z_{1}^{\prime} Q_{(1)}\right) \boldsymbol{Y} . \\
& =\boldsymbol{Y}^{\prime}\left[\frac{1}{|\boldsymbol{S}|}\left(-\lambda_{1,0} Q_{(1)} Q_{(1)}+\lambda_{0,0} Q_{(1)}\left(I_{n q} \otimes J_{p}\right) Q_{(1)}\right)\right] \boldsymbol{Y} .
\end{aligned}
$$

\section{Conclusions}

The conclusions obtained throughout this work are as follows:

1. The MINQUE for $\sigma_{\varepsilon}^{2}$ and $\sigma_{\delta}^{2}$ are

And

$$
\hat{\sigma}_{\varepsilon}^{2}=Y^{\prime}\left[\frac{\left(I_{n}-\left(J_{n} / n\right)\right) \otimes I_{q} \otimes\left(I_{p}-\left(J_{p} / p\right)\right)}{q(n-1)(p-1)}\right] Y
$$

$$
\hat{\sigma}_{\delta}^{2}=Y^{\prime}\left[\frac{\theta\left(I_{n}-\left(J_{n} / n\right)\right) \otimes I_{q} \otimes\left(I_{p}-\left(J_{p} / p\right)\right)}{q(n-1)(p-1)}\right] Y
$$

2. The MINQUE(1) for $\sigma_{\varepsilon}^{2}$ and $\sigma_{\delta}^{2}$ are

And

$$
\hat{\sigma}_{\varepsilon}^{2}=\boldsymbol{Y}^{\prime}\left[\frac{1}{|\boldsymbol{S}|}\left(\lambda_{1,1} Q_{(1)} Q_{(1)}-\lambda_{0,1} Q_{(1)}\left(I_{n q} \otimes J_{p}\right) Q_{(1)}\right)\right] \boldsymbol{Y} .
$$

$$
\hat{\sigma}_{\delta}^{2}=\boldsymbol{Y}^{\prime}\left[\frac{1}{|\boldsymbol{S}|}\left(-\lambda_{1,0} Q_{(1)} Q_{(1)}+\lambda_{0,0} Q_{(1)}\left(I_{n q} \otimes J_{p}\right) Q_{(1)}\right)\right] \boldsymbol{Y} .
$$




\section{References}

[1] Al-Isawi JA. M. A. and AL-Mouel A. H. S. (2018), Best Quadratic Unbiased Estimator for Variance Component of One-Way Repeated Measurement Model, J. Advances in Mathematics, Vol. 14, pp. 7615-7623.

[2] Al-Mouel A. H. S. and Wang, J. L. (2004), One-Way Multivariate Repeated Measurements Analysis of Variance Model. Applied Mathematics a J. Chinese Universities, 19, 4, 435-448.

[3] Al-Mouel A. H. S., Mohaisen A. J. and Khawla (2017), Bayesian One-Way Repeated Measurements Model Based on Bayes Quadratic Unbiased Estimator, Journal of Advance in Mathematics, Vol. 13, No. 2, pp. 7176-7182.

[4] Charles S. Davis (2003), Statistical Methods for the Analysis of Repeated measurements, Springer, Inc. New York.

[5] Eugene D. (2013), Mixed Models Theory and Applications with R, Second Edition, John Wiley \& Sons, Inc., Hoboken, New Jersey.

[6] Graybill F. A. (1983), Matrices with Applications in Statistics, Second Edition, Wadsworth. Inc. USA.

[7] James R. S. (2017), Matrix Analysis for Statistics, Third Edition, John Wiley \& Sons, Inc., Canada.

[8] Rao C. R. and Kleffe J. (1988), Estimation of Variance Components and Applications, North-Holland, Amsterdam.

[9] Rao C. R. (1971a), Estimation of variance and covariance components-MINQUE theory. J. of Multivariate Anal. 1, 257-275.

[10] Rao C. R. (1971b), Minimum variance quadratic unbiased estimation of variance components. J. Multivariate Anal., 1(4), 445-456.

[11] Rao C. R. (1972), Estimation of variance and covariance components in linear models. J. Amer. Statist. Assoc. 67, 112-115.

[12] Sahai H. and Ojeda M. M. (2005), Analysis of variance for random models, Vol. 2, Birkhäuser Boston, USA.

[13] TAN W. Y. (1979), On the Quadratic Estimation of Covariance Matrices in Multivariate Linear Models, J. Multivariate Anal., 9, 452-459.

[14] Vonesh E.F. and Chinchilli V. M. (1997) linear and nonlinear models for the analysis of Repeated Measurements, Marcel Dakker, Inc. New York. 